\title{
Implementation of satellite blood-culture system in an emergency department: impact of time-to results in sepsis detection
}

\author{
Andrea Rocchetti, ${ }^{1}$ Fabio Rapallo, ${ }^{2}$ Paolo Bottino, ${ }^{3}$ Alessandra Mastrazzo² \\ ${ }^{1}$ Laboratory of Microbiology, SS. Antonio e Biagio e C. Arrigo National Hospital, Alessandria; \\ ${ }^{2}$ Department of Science and Technology Innovation, University of Eastern Piedmont, Alessandria; \\ ${ }^{3}$ Department of Medical Science, University of Torino, Italy
}

\section{Summary}

The aim of this study is to compare the time differences in the detection of sepsis, making a comparison between the TAT (turnaround time) of blood cultures in the Emergency Department (ED) compared to the other hospital units. Positive blood cultures were divided into 2 groups: those from ED, and those from other hospital units. For this reason, a continuously monitoring blood culture incubator has been placed in the ED. We considered only adult patients. During the 1-year study, we considered all positive for each patient. Results obtained demonstrate that placing an automated blood culture system in an area of critical importance allows to obtain significant improvements in terms of time and quality of the results. Furthermore, reduction of TAT is determined more by the times of sample transport rather than the processing time.

\section{Introduction}

The fight against sepsis is a clinical priority for healthcare facilities around the world (4). To minimize mortality rates and optimize treat-

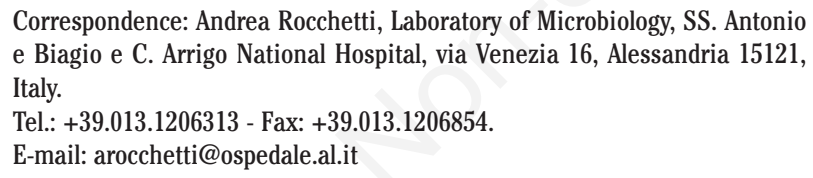

This article is distributed under the terms of the Creative Commons Attribution Noncommercial License (by-nc 4.0) which permits any noncommercial use, distribution, and reproduction in any medium, provided the original author(s) and source are credited. ment, early and accurate detection is a crucial factor $(3,5)$. The Hospital considers Sepsis diagnostic as one of the critical areas that can be improved by optimizing related processes. In 2012 the hospital started a multidisciplinary approach aimed at engaging the entire staff and optimize processes so to i) decrease the time to results ii) decrease time to therapy initiation iii) reduce health-care associated sepsis events by $10 \%$ each year for 3 years.

With this goal in mind a pilot project was initiated between the Microbiology Lab and the adults ED. Besides, more than 30 percent of the total number of sepsis cases is recorded each year at the ED. The shared objectives were i) introduce a near-patient sepsis testing approach; ii) increase staff and patients safety by avoiding bottles to remain unattended prior to incubation iii) reduce time-to results for blood culture testing. One of the variables that impacts the time-to results is the time occurring between sample collection and incubation in the instrument. The time-to incubation can vary substantially during the day, affected generally by the opening hours of the laboratory and other organizational hurdles (staff availability) (3). This can be substantial (up to 6 hours) especially during night shifts and in general during the closing time of the microbiology lab. In the case of Alessandria hospital the microbiology lab is open between 08.00 a.m. and 6.00 p.m from Monday to Saturday and on Sundays there is medical doctor on call. Reducing the time to incubation could hence impact the time to results of the blood cultures and improve quality by avoiding to leave precious samples in sub-optimal conditions.

\section{Type of study}

In order to achieve these goals it was decided to place a BD BAC-

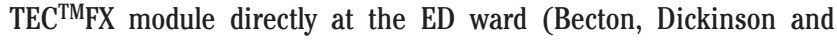
Company, Franklin Lakes, NJ, USA). The entire ED staff was trained on the use of the instrument in order to enter the blood culture bottles immediately after sample collection. The instrument was connected to the data management system (EpiCenter $\left.{ }^{\mathrm{TM}}, \mathrm{BD}\right)$ and to the information system of the hospital. This allowed to monitor remotely from the lab the status of the vials incubated in the ED instrument (satellite system) and monitor pre-analytical, analytical and post-analytical process times. Each morning, at the start of the shift, laboratory staff members would transfer from the ED instrument to the lab all the bottles flagged positive during the night along with those still negatives but from a patient for which one bottle had already turned positive. All the negative bottles moved to the lab instrument were re-incubated within 20 min in order not to lose data integrity and allow the system to continue monitoring the growth. In parallel all the positives blood culture bottles would start the routine work-up for gram-staining, identification and susceptibility testing of the organisms detected. 
Table 1. Turn around time in Emergency Department (ED) vs. other wards. Mean, median and standard deviation (hh:mm) and MannWhitney test.

\begin{tabular}{|c|c|c|c|c|c|c|c|}
\hline & $\begin{array}{l}\text { Me } \\
\text { Satellite ED } \\
\text { incubation }\end{array}$ & $\begin{array}{l}\text { Laboratory } \\
\text { incubation }\end{array}$ & $\begin{array}{l}\text { Mec } \\
\text { Satellite ED } \\
\text { incubation }\end{array}$ & $\begin{array}{l}\text { dian } \\
\text { Laboratory } \\
\text { incubation }\end{array}$ & $\begin{array}{l}\text { Standard d } \\
\text { Satellite ED } \\
\text { incubation }\end{array}$ & $\begin{array}{l}\text { leviation } \\
\text { Laboratory } \\
\text { incubation }\end{array}$ & Mann-Whitney test \\
\hline $\begin{array}{l}\text { Blood collection-incubation } \\
\text { Delta }\end{array}$ & $00: 19$ & $06: 26$ & 00:09 & $02: 18$ & 01:05 & 09:47 & $\mathrm{W}=30,778 ; \mathrm{P}$-value $<2.2 \mathrm{e}-16$ \\
\hline $\begin{array}{l}\text { Gram Staining } \\
\text { Delta }\end{array}$ & 24:28:00 & $2^{37: 52: 00}$ & $21: 33$ & $28^{29: 01: 00}$ & $17: 14$ & $22: 18$ & $\mathrm{~W}=98,602 ; \mathrm{P}$-value $<2.2 \mathrm{e}-16$ \\
\hline $\begin{array}{l}\text { Final Time } \\
\text { Total Delta }\end{array}$ & $\begin{array}{l}\text { 57:01:00 } \\
14:\end{array}$ & $\begin{array}{l}71: 37: 00 \\
36\end{array}$ & $\begin{array}{r}51: 23: 00 \\
14\end{array}$ & :66:17:00 & 24:22:00 & 29:02:00 & $\mathrm{W}=122,691 ; \mathrm{P}$-value $<2.2 \mathrm{e}-16$ \\
\hline
\end{tabular}

This observational study was conducted between 22 February 2013 and 21 February 2014. Various time points (time from blood collection to incubation, time to detection by BACTEC FX, time to Gram-staining, time to final identification and susceptibility testing)of samples incubated directly at the ED (satellite instrument) were monitored and compared to samples collected from other wards and incubated in the laboratory instrument as per standard routine.

Data collected were processed using the statistical software R. For each different time of TAT Mean, Standard Deviation and Median were evaluated. For assessing the statistical significance of the differences of the two distributions, we have used the nonparametric MannWhitney test and a P-value less than 0.05 was considered significant (Table 1).

\section{Conclusions}

During this period 1084 blood cultures were executed at the ED and 4631 at the remaining wards. The satellite approach at the ED yielded an immediate reduction of $6 \mathrm{~h}$ of waiting time occurring between the sample collection and the instrument incubation. In the ED, the average time from sample collection to Gram-staining was $24 \mathrm{~h}: 28$ ' $37 \mathrm{~h}: 52$ ' recorded for the other wards. This accounted for $13 \mathrm{~h}: 24$ ' reduction for the ED results compared to the other wards. This saving in time seems to suggest that immediate incubation of the sample contributes to a faster growth of the organisms in the blood culture vials (3). The time until final results for the ED is an average of 57 hours, 14 hours faster than the other departments (Table 1)

Implementation of the satellite approach, with immediate incubation of blood samples yielded a significant improvement in diagnostic times and allowed the Microbiology Laboratory to communicate impor- tant information to the clinicians in order to move from an empirical therapy to a more targeted one (1).

This short evaluation proved that with limited investment in terms of equipment or technologies it is possible to optimize the diagnostics processes and facilitates clinical decision making (2). Limitation of this evaluation are the limited number of data collected and the scope restricted to the hospital. It would be interesting to further prove the positive impact of immediate incubation extending the satellite approach to other critical wards.

Another limitation is that further studies are needed to investigate how this improvement can help the clinician to determine the impact on patient survival, following antibiotic shift in therapy.

\section{References}

1. Barenfanger J, Drake C, Kacich G. Clinical and financial benefits of rapid bacterial identification and antimicrobial susceptibility testing. J Clin Microbiol 1999;37:1415-8.

2. Yealy D, Huang D, Delaney A, et al. Recognizing and managing sepsis: what needs to be done?. BMC Med 2015;13:98.

3. Kerremans J, van der Bij A, Goessens W, et al. Immediate incubation of blood cultures outside routine laboratory hours of operation accelerates antibiotic switching. J Clin Microbiol 2009;47:3520-3.

4. Rivers E, Nguyen B, Havstad S, et al. Early goal-directed therapy in the treatment of severe sepsis and septic shock. N Engl J Med 2001;345:1368-77.

5. Vallés J, Rello J, Ochagavía A, et al. Community-acquired bloodstream infection in critically ill adult patients. Chest 2003;123: 1615-24. 